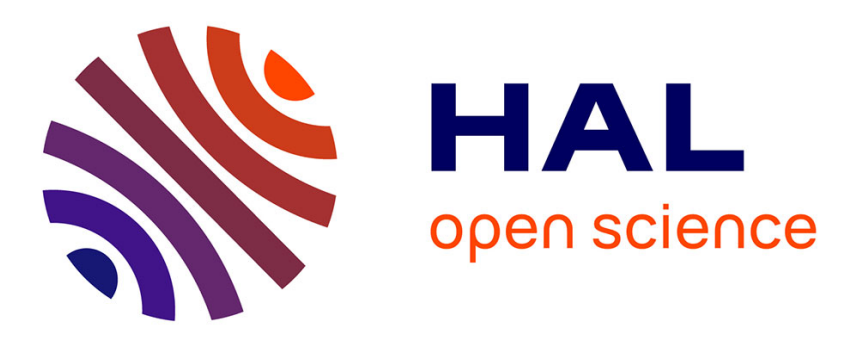

\title{
Human arm optimal motion analysis in industrial screwing task
}

Nahéma Sylla, Vincent Bonnet, Gentiane Venture, Nasser Armande, Philippe Fraisse

\section{- To cite this version:}

Nahéma Sylla, Vincent Bonnet, Gentiane Venture, Nasser Armande, Philippe Fraisse. Human arm optimal motion analysis in industrial screwing task. BioRob: Biomedical Robotics and Biomechatronics, Aug 2014, São Paulo, Brazil. pp.964-969, 10.1109/BIOROB.2014.6913905 lirmm-01111274

\section{HAL Id: lirmm-01111274 https://hal-lirmm.ccsd.cnrs.fr/lirmm-01111274}

Submitted on 30 Jan 2015

HAL is a multi-disciplinary open access archive for the deposit and dissemination of scientific research documents, whether they are published or not. The documents may come from teaching and research institutions in France or abroad, or from public or private research centers.
L'archive ouverte pluridisciplinaire $\mathbf{H A L}$, est destinée au dépôt et à la diffusion de documents scientifiques de niveau recherche, publiés ou non, émanant des établissements d'enseignement et de recherche français ou étrangers, des laboratoires publics ou privés. 


\title{
Human arm optimal motion analysis in industrial screwing task
}

\author{
N. Sylla ${ }^{1,3}$, V. Bonnet ${ }^{2}$, G. Venture ${ }^{2}$, N. Armande ${ }^{1}$ and P. Fraisse ${ }^{3}$
}

\begin{abstract}
In this study, we propose to explore inverse optimization process to better understand human arm motion in industrial screwing task. The process combines several criteria to minimize such as energy expenditure or trajectory smoothness leading to the optimal trajectory of a typical screwing task, often performed by workers. Estimated joint trajectories are similar with the measured ones, with a mean square error of 4 degrees. The resulting cost-function is mainly composed of energy expenditure and geodesic criteria. Results show the relevance of using composite cost function in human motion planning. This study has been conducted to assist workers by using collaborative robots in painful task in PSA Peugeot Citroen factories to improve ergonomics of manual workstations.
\end{abstract}

\section{INTRODUCTION}

In car production lines, although numerous processes have been automated, some operations remain manual mainly because of reduced workspaces and precision requirements. However, workers dedicated to these jobs could be subjected to adopt awkward postures and carry notable efforts. PSA Peugeot Citroen aims to use collaborative robots (cobots), which present the functionality to work together with humans for ergonomic assistance [1] to improve occupational health and prevent musculoskeletal disorders. According to their reachability and their number of degrees of freedom (DoFs), cobots could be very expensive and not adapted to all tasks. Furthermore, ergonomic analysis methods currently used in industries are unable to find out these limitations since they are subjective, principally based on movement observation [2], and rarely consider biomechanics of movements. Consequently, it is necessary to determine criteria involved in workers' movements behaviour. This approach is relevant (1) to improve ergonomic assessment of industrial tasks, (2) to help in determining the optimal assistive device to implement according to workers movements.

According to the literature in human motor control since the last 30 years, human movements are controlled by the central nervous system (CNS), which selects the optimal motion strategy among several solutions before sending the information to the musculoskeletal system [3]. However, the choice of this optimal trajectory is not trivial. The human

\footnotetext{
${ }^{1}$ PSA Peugeot Citroen Research and Development Department, Centre Technique de Vlizy, Route de Gisy, 78140 Velizy Villacoublay, France, khadijanahema.sylla@mpsa.com, nahid.armande@mpsa.com

${ }^{2} \mathrm{GV}$ laboratory, Dpt. of Mechanical Systems Engineering, Tokyo University of Agriculture and Technology, Tokyo University, Japan vincent.bonnetegmail.com, ventureecc.tuat.ac.jp

${ }^{3}$ LIRMM (Montpellier laboratory of Informatics, Robotics and Micro-electronics), 161 rue Ada, 34392 Montpellier Cedex 5, France fraisselirmm.fr
}

musculoskeletal system often presents more DoFs than the ones necessary to accomplish a specific movement. This kinematic, dynamic and actuation redundancy issue is not straightforward in term of motion equations. Recently, observations in human motion science together with progresses in computational power, optimization and new algorithms coming from humanoid robotics field have allowed to assess this problematic over more and more complex tasks.

Numerous cost functions have been identified in the literature. Flash and Hogan proposed to minimize a purely kinematic quantity, the cartesian jerk [4] defined as the sum of the square of the third derivative of cartesian coordinates, for an arm movement in the horizontal plane only.

The minimum torque change criterion which corresponds to the sum of human joint torques first derivatives, was proposed by Uno et al. [5]. The study used a 2 joints arm model, allowing movements in the horizontal plane. However results were not validated for 3D movements.

The minimum energy criterion was presented by Alexander et al. [6] with a 2 DoFs arm model, and was used later by Taix et al. [7] for reaching tasks performed by the 6 DoFsarm of an humanoid robot. Several others criteria such as minimum work [8], minimum variance [9], minimum joint constraint forces [10], or minimum time [11] have been proposed for multi-joints movements. From this literature no consensus emerges and it appears that each of these optimal criteria accounts for a feature of the motion depending of the considered population and/or task.

The use of hybrid cost-functions was then introduce to determine optimal trajectories. Otha et al. [12] proposed to combine the hand force change and torque change criteria to determine the optimal trajectory of human arm movements in crank rotation tasks. Following this work, the inverse optimal process was proposed by Monbaur et al. [13] to determine optimality criteria that produce natural paths for human locomotion. Resulting data were applied in humanoid robots path planning. The human body was considered as a single point and experiments were performed in a $2 \mathrm{D}$ plane. These hypotheses simplified significantly the inverse optimization process. Albretch et al. [14] also performed inverse optimization considering properties of both skeletal and muscular system of the human arm. The retained costfunction combining cartesian jerk, torque change, joint jerk and muscle tension change was applied on an only $2 \mathrm{DoFs}$ arm model. Later Berret et al. proposed a similar approach to obtain the best cost function which characterizes a reachinga-bar movement using a 2 DoFs arm model [15]. However this interesting and well documented study focuses on a simple planar task with a poorly constrained paradigm. 
To the best of our knowledge, no study uses the inverse optimal process for complex 3D tasks and/or industrial purpose. The present study focuses on an under-car workstation which is very challenging in terms of ergonomics. According to PSA Peugeot Citroen ergonomicists measures, the main movement is repeated 35\% times in a cycle of 110 seconds approximately. It consists of raising the arm holding on a screw-gun to reach a point located 2 meters above the ground. Based on studies presented in [13] and [15], an inverse optimization process is used to determine the optimal hybrid criterion that best describes this screwing movement. The arm model presents 4 DoFs allowing movement in $3 \mathrm{D}$ plane.

The paper is organised as follows: Section II describes the experiment protocol and measurements. The arm model and the inverse optimization process are presented in Section III, and Section IV discusses the obtained results.

\section{EXPERIMENTATION}

\section{A. Experimental setup and measurements}

Eight right-handed volunteers not familiar with the task reproduced the typical screwing movement. Starting from an initial standing position with both hands along the body side, they were asked to reach a target located 2 meters above the ground, holding on a $0.95 \mathrm{~kg}$ screwgun in their right hand (see Fig. 1). No particular instruction was indicated to the subjects and the movement was performed in their most natural way, at their preferred velocity.

3D kinematic quantities were recorded using a motion capture system (6 MX cameras, VICON, $100 \mathrm{~Hz}$ ) in combination with a set of 38 retro-reflective markers. Markers were placed on anatomical landmarks in accordance with the Plug-inGait whole body marker template (Vicon Motion Systems) to reconstruct joint angles.

\section{B. Human observations}

Averages and standard deviations of subjects' joint angles were normalized to 100 data points and reported in Fig.

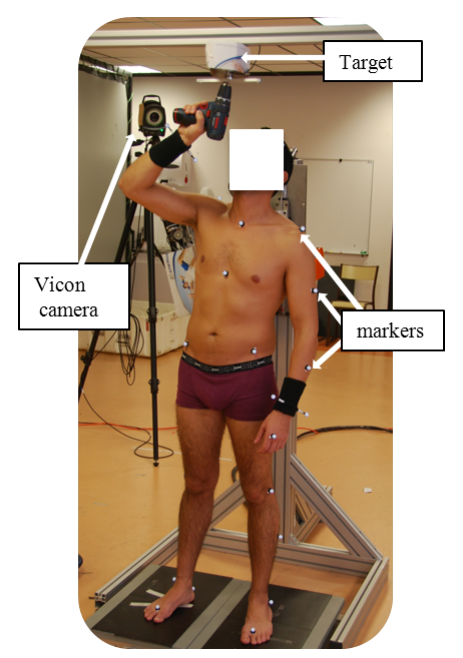

Fig. 1. The investigated screwing task.
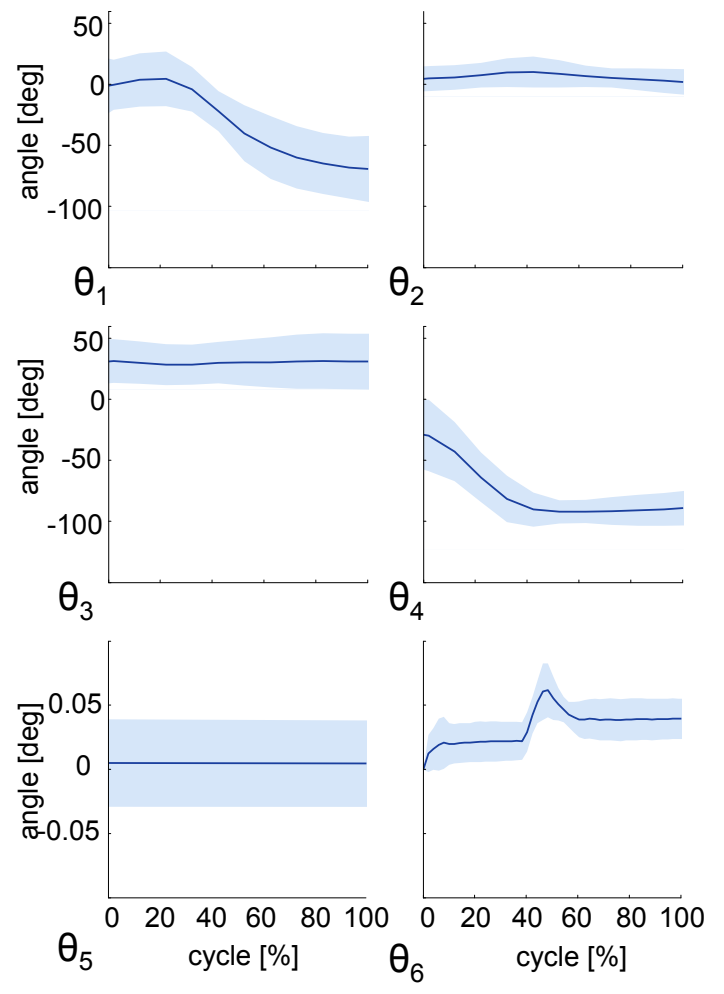

Fig. 2. Averages and standard deviations of measured shoulder flexion/extension $\left(\theta_{1}\right)$, shoulder abduction/adduction $\left(\theta_{2}\right)$, forearm pronation/supination $\left(\theta_{3}\right)$, elbow flexion/extension $\left(\theta_{4}\right)$, wrist flexion/extension $\left(\theta_{5}\right)$ and wrist abduction adduction $\left(\theta_{6}\right)$.

2. Note that the observed task results in low amplitude of shoulder movements in the transverse plane. As expected measured values of shoulder rotation were negligible and were not reported in Fig. 2. $\theta_{1}, \theta_{2}, \theta_{3}, \theta_{4}, \theta_{5}$ and $\theta_{6}$ corresponds respectively to shoulder flexion/extension, shoulder abduction/adduction, forearm pronation/supination, elbow flexion/extension, wrist flexion/extension and wrist abuduction/adduction. From this figure one can see the relatively low standard deviation of joint angles, showing that subjects perform similar and consistent movements to reach the target. Furthermore, the observed wrist joints range of motion are very small. Consequently, wrist joints are neglected in the human arm model.

\section{MODELING OF THE SCREWING TASK}

\section{A. Human arm model}

The retained human arm model is composed of 4 DoFs, represented in Fig. 3. Joint torques are computed from Lagrangian formulation of the inverse dynamical model of the arm [16]:

$$
\Gamma=A(\theta) \ddot{\theta}+C(\theta, \dot{\theta}) \dot{\theta}+Q(\theta),
$$

where $\Gamma=\left[\begin{array}{llll}\Gamma_{1} & \Gamma_{2} & \Gamma_{3} & \Gamma_{4}\end{array}\right]^{T}$ is the vector of joint torques, $A(\theta), C(\theta, \dot{\theta})$ and $Q(\theta)$ are respectively the inertial matrix, the Coriolis and Centrifugal effect matrix, and the gravity matrix computed from human bodyweight, eight, and joint angles. $\boldsymbol{\theta}$ corresponds to the vector of joint angles: 


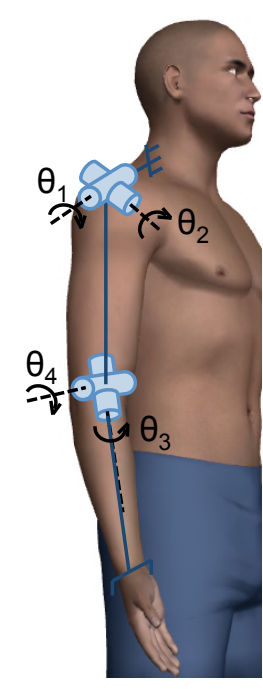

Fig. 3. The human arm kinematic model in its zero-configuration.

$\boldsymbol{\theta}=\left[\begin{array}{llll}\theta_{1} & \theta_{2} & \theta_{3} & \theta_{4}\end{array}\right]^{T}$.

\section{B. Direct optimization}

The direct optimization process consists of finding the optimal value of designated parameters by minimizing an objective cost-function while respecting all listed constraints. Applying this principle to our study, direct optimization will generate the best values of joint angles that produce the minimum value of a given criterion. Table I reports mathematical expressions of all criteria used in this study. Criteria were normalized by their corresponding value computed from measured human movement data. When performing optimization by using each cost function independently, resulting joint angles are very different with the measured ones (see Fig. 4). Combining these cost functions is then relevant to obtain better results [15].

The retained hybrid cost function includes then all criteria:

$$
J=\sum_{i=1}^{7} \alpha_{i} C_{i}
$$

where $\alpha_{i}$ are the positive weights that define the contribution of every criterion $C_{i}$ [15], normalized according to the following equation:

$$
C_{i}=\frac{c_{i}}{c_{r e f}(i)},
$$

where $c_{i}$ corresponds to the criterion as defined in Table I, and $c_{r e f}(i)$ its corresponding value when the movement is performed by subjects, i.e. using measured subjects joint angles, torques, and end effector position values: $c_{r e f}=$

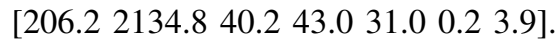

Joint angles data vectors and their corresponding derivatives were sampled using a $5^{\text {th }}$ order spline interpolation. Preliminary studies were performed to determine the optimal number of knots. 12 knots appeared to be the good trade-off between the number of parameters that need to be identified and the accuracy in reproducing joint trajectories.

Constraints imposed for the experimentation are listed below:

- Angular limits: To generate feasible motions, optimized trajectories must be compliant with human angular limits defined in the literature [2]:

$$
\boldsymbol{\theta}_{\text {min }} \leq \boldsymbol{\theta} \leq \boldsymbol{\theta}_{\text {max }},
$$

where $\boldsymbol{\theta}_{\min }$ and $\boldsymbol{\theta}_{\max }$ are respectively vectors of minimum and maximum of human joint angles reported in Table II.

- Final position and orientation: Regarding the defined arm model (Fig. 3), the point to reach corresponds to the final position of the right hand. Final human arm orientation is also given as a constraint. Right hand position and orientation are deducted from the direct geometric model [16] of the human arm developed in Matlab using symbolic approach.

TABLE I

COST FUNCTION USED IN THE STUDY [15]

\begin{tabular}{|c||c|}
\hline Criterion & Cost function ${ }^{a}$ \\
\hline Cartesian jerk & $c_{1}=\frac{\sum_{j=1}^{n} \dddot{x}_{j}^{2}+\dddot{y}_{j}^{2}+\dddot{z}_{j}^{2}}{n}$ \\
Angle jerk & $c_{2}=\frac{\sum_{j=1}^{n} \sum_{i=1}^{4} \dddot{\theta}_{i_{j}}^{2}}{n}$ \\
Angle acceleration & $c_{3}=\frac{\sum_{j=1}^{n} \sum_{i=1}^{4} \ddot{\theta}_{i_{j}}^{2}}{n}$ \\
Torque change & $c_{4}=\frac{\sum_{j=1}^{n} \sum_{i=1}^{4} \dot{\Gamma}_{i_{j}}^{2}}{n}$ \\
Torque & $c_{5}=\frac{\sum_{j=1}^{n} \sum_{i=1}^{4} \Gamma_{i_{j}}^{2}}{n}$ \\
Geodesic & $c_{6}=\frac{\sum_{j=1}^{n} \sqrt{\dot{\boldsymbol{\theta}}_{j}^{T} \boldsymbol{A}(\boldsymbol{\theta}) \dot{\theta}_{j}}}{n}$ \\
Energy & $c_{7}=\frac{\sum_{j=1}^{n} \sum_{i=1}^{4}\left|\dot{\theta}_{i_{j}} \Gamma_{i_{j}}\right|}{n}$ \\
\hline
\end{tabular}

a. $n$ is the length of joint angles and positions variables

TABLE II

ANGULAR LIMITS

\begin{tabular}{|c||c||c|}
\hline Joint angle & $\theta_{\min }[\mathrm{deg}]$ & $\theta_{\max }[\mathrm{deg}]$ \\
\hline$\theta_{1}$ & -180 & 55 \\
$\theta_{2}$ & -150 & 30 \\
$\theta_{3}$ & -100 & 100 \\
$\theta_{4}$ & -160 & 0 \\
\hline
\end{tabular}



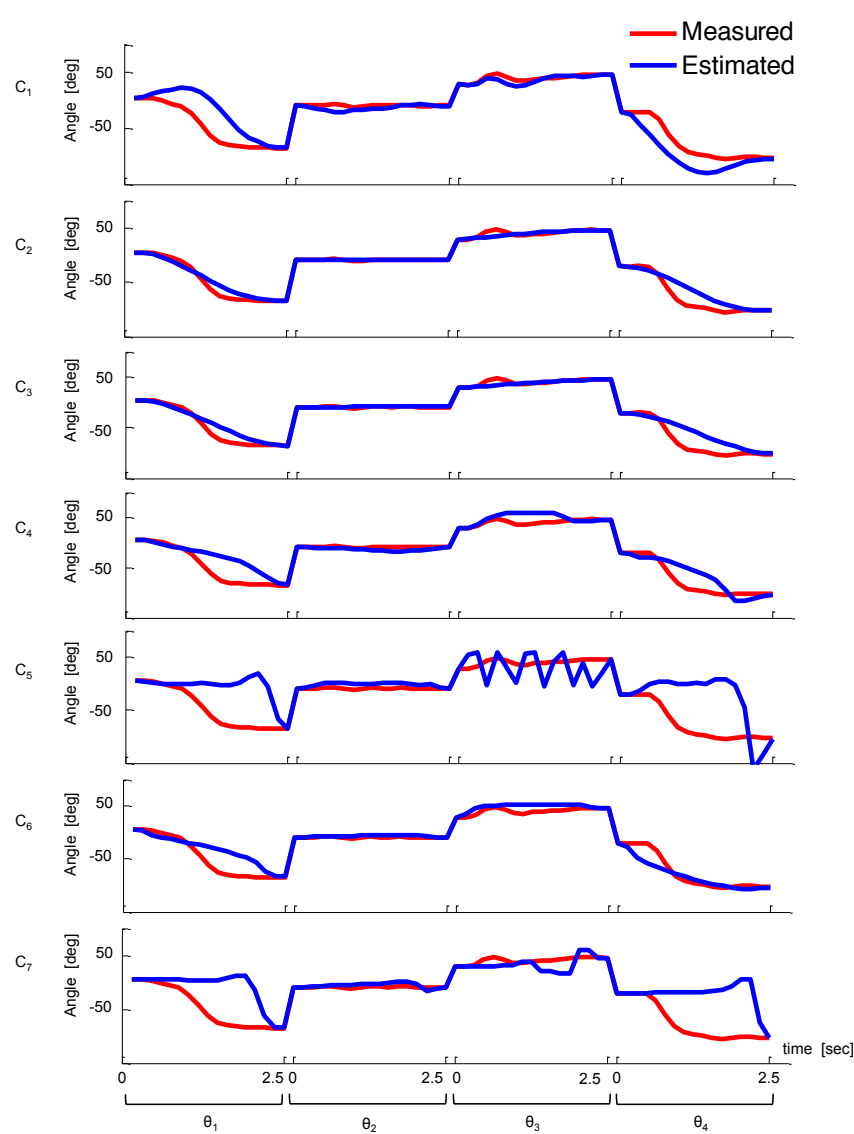

Fig. 4. Comparison of measured and estimated joint angles when using independently one of the normalized criteria $\left(C_{i}\right)$ presented in Table I.

\section{Inverse optimization}

The aim of the inverse optimization process is to determine the best combination of $\boldsymbol{\alpha}$ weights that will minimise the root mean square error (RMSE) between estimated $\left(\boldsymbol{\theta}_{\text {est }}\right)$ and measured $\left(\boldsymbol{\theta}_{\text {mes }}\right)$ joint angles.

Inverse optimization is composed by two interlocked optimization processes. The first one is an outer optimization which minimizes the RMSE between measured and estimated joint angles, and the second one is an inner optimization which minimizes the cost function $J$.

Fig. 5 is an outline of the computational procedure used for inverse optimization. The variable $\Delta$ corresponds to RMSE between measured and estimated joint angles:

$$
\boldsymbol{\Delta}=\sqrt{\frac{\sum_{j=1}^{n}\left(\boldsymbol{\theta}_{\boldsymbol{m e s}_{\boldsymbol{j}}}-\boldsymbol{\theta}_{\boldsymbol{e s t}_{\boldsymbol{j}}}\right)^{2}}{n}},
$$

where $n$ is the length of $\boldsymbol{\theta}_{\text {mes }}$ and $\boldsymbol{\theta}_{\text {est }}$ vectors.

Changes of $\boldsymbol{\alpha}$ weights are performed by the algorithm. The program written in Matlab includes then two optimization processes. The first one, which determines the optimal set of $\boldsymbol{\alpha}$ weights is performed with a genetic algorithm [17] due to the large scale of the optimization problem. The second one, which determines optimal joint angle trajectories

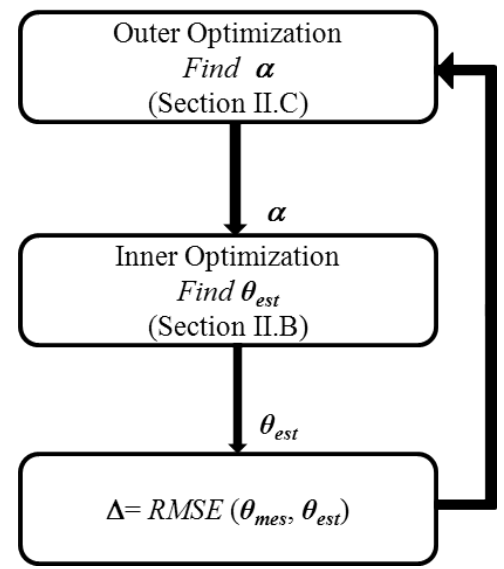

Fig. 5. Outline of the computational procedure used for inverse optimization.

is performed using a gradient based non linear optimization algorithm [18].

\section{RESULTS}

Fig. 6 and 7 show representative results obtained with one subject. It can be seen that joint angles and cartesian trajectories obtained with the optimization process are fairly similar to the measured ones. The resulting RMSE vector between estimated and measured joint angles is (in degrees): $\Delta=\left[\begin{array}{lllll}4 & 1.1 & 4.4 & 6.8\end{array}\right]^{T}$. Values of normalized weights obtained by inverse optimization are the following: $\alpha=\left[\begin{array}{llll}0.3 & 1.0 & 0.1 & 0.0\end{array}\right.$ $0.05 .25 .8]^{T}$.

Fig. 8 shows the contribution in $\%$ of every weighted cost function in the total hybrid cost function $J$. From these results one can see low contributions of jerk, angle acceleration, torque change and torque criteria during the movement. The energy is as expected the most important criterion with a contribution of $53.7 \%$ in the resulting cost function. The second cost function mainly involved in the screwing movement is the geodesic one. This criterion was introduced by Biess et al. [19] [20], and has been validated in their study with a $3 \mathrm{D}$ point-to point movement with a 4 DoFs arm model. It combines geometrical (e.g. hand path and arm posture) and temporal features of a movement. The Geodesic criterion corresponds to the shortest path in the curved space. In our case, it means: the shortest path in the joint space. In term of energy, it amounts to the minimum energy of the curve.

Results presented in Berret et al. study [15] showed that the energy is minimized and joints smoothness is maximized during a reaching-a-bar task. The resulting cost-function combine energy and angle acceleration criteria hence. Knowing that the joint smoothness maximization is also included in the geodesic criterion, our results are in accordance with the previous one. We can then conclude that workers minimize their energy expenditure, the path distance in the joint space while maximizing arm joints smoothness when performing the screwing task. 


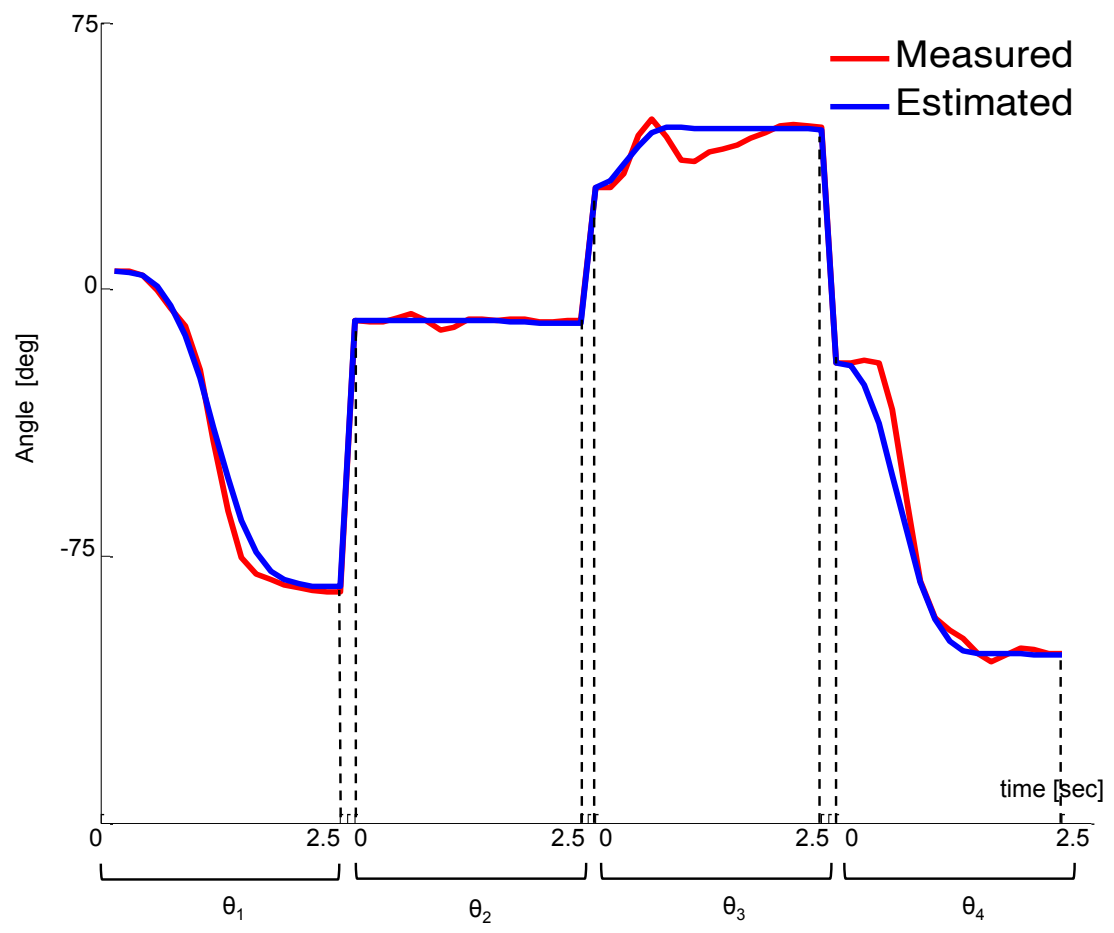

Fig. 6. Measured and estimated joint angles of a typical subject.

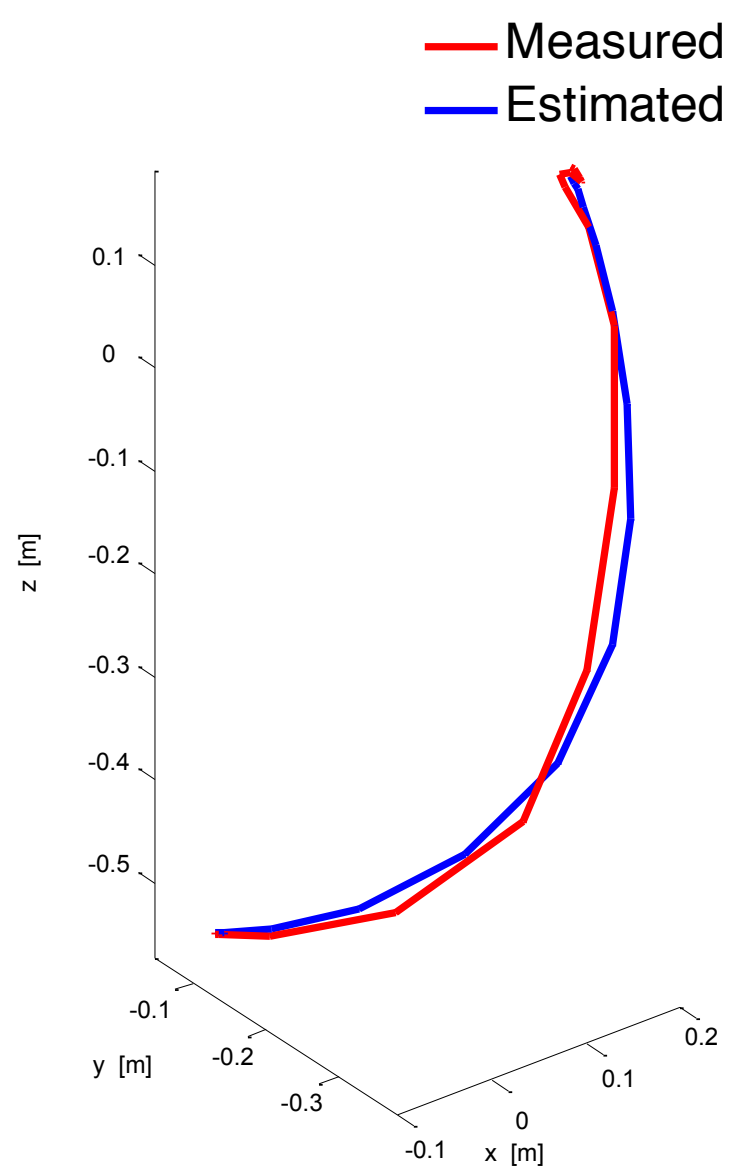

Fig. 7. Cartesian trajectories of measured and estimated movement.

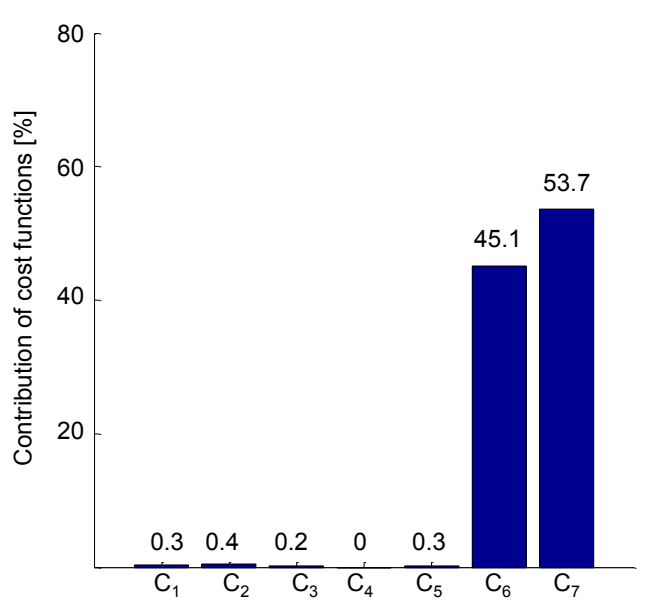

Fig. 8. Contribution in $\%$ of each weighted cost function to the total hybrid cost function.

\section{DISCUSSION}

For a representative subject, results show a RMSE average of 4 degrees between measured joint angles and the estimated ones through the optimization process while respecting joint angles averages represented in Fig. 2, and justify in this case the use of hybrid cost function. Regarding the investigated screwing task, high contributions of energy and geodesic criteria in the resulting cost-function are relevant.

Further analysis with other subjects is being performed 
to validate the hybrid cost function obtained here. It is important to note that inverse optimization requires high computational power. One analysis takes about 30 hours of simulations on a $2.70 \mathrm{GHz}$ Intel core i7.

\section{CONCLUSION}

In this study we investigated inverse optimization process [15] to determine criteria that are minimized when performing a typical under-car screwing task, which is very challenging in terms of ergonomics and appears to cause muskuloskeletal disorders. No solution has been proposed yet to assist workers in this task which is difficult to automate. By understanding human mechanisms involved during the screwing movement from these results, we believe that we will be able to determine the optimal assistive device in terms of DoFs and command strategy to improve workers' comfort. Slight differences between measured joint angles and the estimated ones show the relevance of using a hybrid cost function in human motion planning. Results have to be consolidated for all subjects, and future experiments will be carried out with experimented workers in factory to confirm these preliminary results.

\section{REFERENCES}

[1] P. Akella, M. Peshkin, E. Colgate, W. Wannasuphoprasit, N. Nagesh, J. Wells, S. Holland, T. Pearson, and B. Peacock, "Cobots for the automobile assembly line," in Proceedings, IEEE International Conference on Robotics and Automation, vol. 1, pp. 728-733, IEEE, 1999.

[2] V. Hue, Simulation de mouvement humain sur postes de travail pour le diagnostic et l'aide à la conception. $\mathrm{PhD}$ thesis, Université Toulouse II Paul Sabatier, Oct. 2008.

[3] S. H. Scott, "Optimal feedback control and the neural basis of volitional motor control," Nature Reviews Neuroscience, vol. 5, no. 7, pp. 532-546, 2004.

[4] T. Flash and N. Hogan, "The coordination of arm movements: An experimentally confirmed mathematical model," The Journal of Neuroscience, vol. 5, pp. 1688-1703, July 1985 .

[5] Y. Uno, M. Kawato, and R. Suzuki, "Formation and control of optimal trajectory in human multijoint arm movement," Biological Cybernetics, vol. 61, no. 2, pp. 89-101, 1989.

[6] R. M. Alexander, "A minimum energy cost hypothesis for human arm trajectories," Biological Cybernetics, vol. 76, pp. 97-150, Feb. 1997.

[7] M. Taïx, M. T. Tran, P. Soueres, and E. Guigon, "Generating humanlike reaching movements with a humanoid robot: A computational approach," Journal of Computational Science, vol. 4, pp. 269-284, July 2013.

[8] T. Kang, J. He, and S. I. H. Tillery, "Determining natural arm configuration along a reaching trajectory," Experimental Brain Research, vol. 167, no. 3, pp. 352-361, 2005.

[9] C. Harris and D. Wolpert, "Signal-dependent noise determines motor planning. [nature. 1998] - PubMed - NCBI," Nature, vol. 394 no. 6695 , pp. $780-784,1988$.

[10] R. Maas and S. Leyendecker, "Optimal control of biomechanical motion using physiologically motivated cost functions," in The 2nd joint international conference on multibody system dynamics, 2012.

[11] W. L. Nelson, "Physical principles for economies of skilled movements - springer," Biological Cybernetics, vol. 46, no. 2, pp. 135-147, 1993.

[12] K. Ohta, M. M. Svinin, Z. Luo, S. Hosoe, and R. Laboissiere, "Optimal trajectory formation of constrained human arm reaching movements,' Biological cybernetics, vol. 91, no. 1, pp. 23-36, 2004.

[13] K. Mombaur, A. Truong, and J. P. Laumond, "From human to humanoid locomotion - an inverse optimal control approach," Autonomos Robots, vol. 28, no. 3, pp. 369-383, 2010.

[14] S. Albrecht, M. Sobotka, and M. Ulbrich, "A bilevel optimization approach to obtain optimal cost functions for human arm-movements,' Preprint in preparation, Fakultät für Mathematik, TU München, 2010.

[15] B. Berret, E. Chiovetto, F. Nori, and T. Pozzo, "Evidence for composite cost functions in arm movement planning: An inverse optimal control approach," PLoS Computational Biology, vol. 7, pp. 1-18, Oct. 2011.

[16] W. Khalil and E. Dombre, Modeling, identification and control of robots. hermes ed., 1999.

[17] C. R. Houck, J. A. Joines, and M. G. Kay, "A genetic algorithm for function optimization: a matlab implementation," NCSU-IE TR, vol. 95, no. 09, 1995.

[18] R. H. Byrd, J. C. Gilbert, and J. Nocedal, "A trust region method based on interior point techniques for nonlinear programming," Mathematical Programming, vol. 89, no. 1, pp. 149-185, 2000.

[19] A. Biess, M. Nagurka, and T. Flash, "Simulating discrete and rhythmic multi-joint human arm movements by optimization of nonlinear performance indices," Biological cybernetics, vol. 95, no. 1, pp. 31-53, 2006.

[20] A. Biess, D. G. Liebermann, and T. Flash, "A computational model for redundant human three-dimensional pointing movements: Integration of independent spatial and temporal motor plans simplifies movement dynamics," The Journal of Neuroscience, vol. 27, pp. 13045-13064, Nov. 2007. 